\title{
Squamous Cell Carcinoma of the Temporal Bone Arising from Cholesteatoma: A Case Report and Review of the Literature
}

\author{
Juan C. Yanez-Siller ${ }^{1} \quad$ Carissa Wentland $^{1} \quad$ Kelly Bowers $^{2} \quad$ N. Scott Litofsky ${ }^{3} \quad$ Arnaldo L. Rivera $^{1}$
}

${ }^{1}$ Department of Otolaryngology-Head and Neck Surgery, University of Missouri, Columbia, Missouri, United States

2 Department of Pathology and Anatomical Sciences, University of Missouri, Columbia, Missouri, United States

${ }^{3}$ Division of Neurosurgery, Department of Surgery, University of Missouri, Columbia, Missouri, United States
Address for correspondence Arnaldo L. Rivera, MD, Department of Otolaryngology-Head and Neck Surgery, University of Missouri, 1 Hospital Dr, MA314, Columbia, MO 65212, United States (e-mail: riveraa@health.missouri.edu).

J Neurol Surg Rep 2022;83:e13-e18.

\begin{abstract}
Keywords

- squamous cell carcinoma of temporal bone

- cholesteatoma

- skull base

- chronic otorrhea

Objective Present a case of squamous cell carcinoma of the temporal bone (SCCTB) arising in a 61-year-old female with a prior history of cholesteatoma and persistent otologic symptoms and review the current literature regarding this disease presentation.

Setting Tertiary academic center.

Patient A 61-year-old female with a history of left ear cholesteatoma for which she had undergone surgery 54 years prior. The patient presented with a persistent history of otorrhea since first surgery and developed exacerbation of symptoms just prior to presentation at our department. The clinical picture was highly suspicious of cholesteatoma recurrence. However, the biopsy was consistent with squamous cell carcinoma.

Intervention Surgical debulking of the lesion was followed by a brief course of radiation therapy later halted by the patient due to side effect intolerance.

Conclusion SCCTB may arise from cholesteatoma. A high index of suspicion for SCCTB should be maintained in patients with a prior history of cholesteatoma and evidence of a temporal bone mass with persistent otologic symptoms.
\end{abstract}

\section{Introduction}

Cholesteatoma is a relatively common benign disease of the middle ear. By contrast, squamous cell carcinoma of the temporal bone (SCCTB) is a rare diagnosis and has a relatively poor prognosis, particularly in advanced stages. ${ }^{1}$ A few reports have implicated cholesteatoma as an etiologic factor for SCCTB. ${ }^{2-5}$ However, a direct association between both entities (i.e., progression from cholesteatoma to squamous cell carcinoma $[\mathrm{SCC}]$ ) remains unproven.

received

October 4, 2020

accepted

April 14, 2021
DOI https://doi.org/ 10.1055/s-0041-1741069. ISSN 2193-6358.

\section{Case Report}

We present a case of SCCTB in a 61-year-old female who had undergone surgery for a left ear cholesteatoma 54 years prior. The patient presented to our institution with a long history of left-side hearing loss, persistent ipsilateral otorrhea, and vertigo. All symptoms had reportedly been present since just after her first ear surgery but had intensified a few months before the latest encounter. She had no personal or significant family history of malignancy. Physical

\footnotetext{
(c) 2022. The Author(s).

This is an open access article published by Thieme under the terms of the Creative Commons Attribution-NonDerivative-NonCommercial-License, permitting copying and reproduction so long as the original work is given appropriate credit. Contents may not be used for commercial purposes, or adapted, remixed, transformed or built upon. (https://creativecommons.org/ licenses/by-nc-nd/4.0/) Georg Thieme Verlag KG, Rüdigerstraße 14, 70469 Stuttgart, Germany
} 
examination revealed tympanic membrane perforation, purulent otorrhea, and mild canal edema with granulation tissue. No mastoid tenderness or fluctuance was present.

A computed tomography scan was significant for an expansile left temporal bone (TB) lesion and tegmen erosion. A subsequent magnetic resonance imaging (MRI) scan lesion extension into the dura overlying the left cerebellar hemisphere. No evidence of lymphadenopathy was present on imaging or physical examination. While the clinical picture was highly suspicious of cholesteatoma recurrence, imaging was concerning for malignancy. Thus, the decision was made to proceed to the operating room jointly with neurosurgery for a combined left middle cranial fossa (MCF) approach and mastoidectomy for biopsy with possible total resection.

Neurosurgery performed a MCF approach. During osteoplastic flap elevation, the lesion was noted to involve the bony flap and the lateral skull base. The lesion was probed and was noted to extend into the left jugular bulb, sigmoid sinus, and posterior fossa dura, as well as to be continuous with the skin of the external acoustic canal (EAC). The facial nerve and vestibule were both uninvolved. A small portion was sent for intraoperative pathology consultation. Diff-Quikstained squash preparation demonstrated numerous dispersed and cohesive squamous cells with marked nuclear atypia and the hematoxylin and eosin stained histology slide confirmed SCC. Given the extent of the disease and the low likelihood of improving the patient's prognosis with aggressive TB resection, only conservative lesion debulking, with preservation of the EAC, was performed. The remainder of the excised tissue was submitted for permanent pathologic evaluation. Microscopic examination revealed invasive keratinizing well-differentiated SCC that invades the stroma and bone, with adjacent areas of dysplastic squamous epithelium (see - Fig. 1A-D). Additionally, there were focal areas with "flaky" keratin debris, fibrosis, and chronic inflammation, suggestive of a cholesteatoma (see - Fig. 1E-F). For research purposes, human papillomavirus (HPV) testing was performed on the tissue sample. There was no staining with p16 immunostain, and HPV DNA in situ hybridization for family 6 (types 6 and 11) and family 16 (types 16, 18, 31, 33, and 51) was negative.

A postoperative positron emission tomography scan revealed no evidence of distant metastasis or another source of primary focus. Following the University of Pittsburgh Staging System, the final pathologic stage was pT4 pNO $\mathrm{PM} 0$, given the TB and dural involvement. Adjuvant radiotherapy was then pursued; however, it was halted early due to side effect intolerance by the patient. The patient opted to undergo palliation treatment instead. The patient was still alive at the time of submission.

\section{Discussion}

Primary SCCTB is a rare condition, with an estimated one to six cases per 1 million population per year worldwide and comprising less than $0.2 \%$ of all head and neck tumors. ${ }^{6,7}$ However, of the different types of tumors to involve the TB,

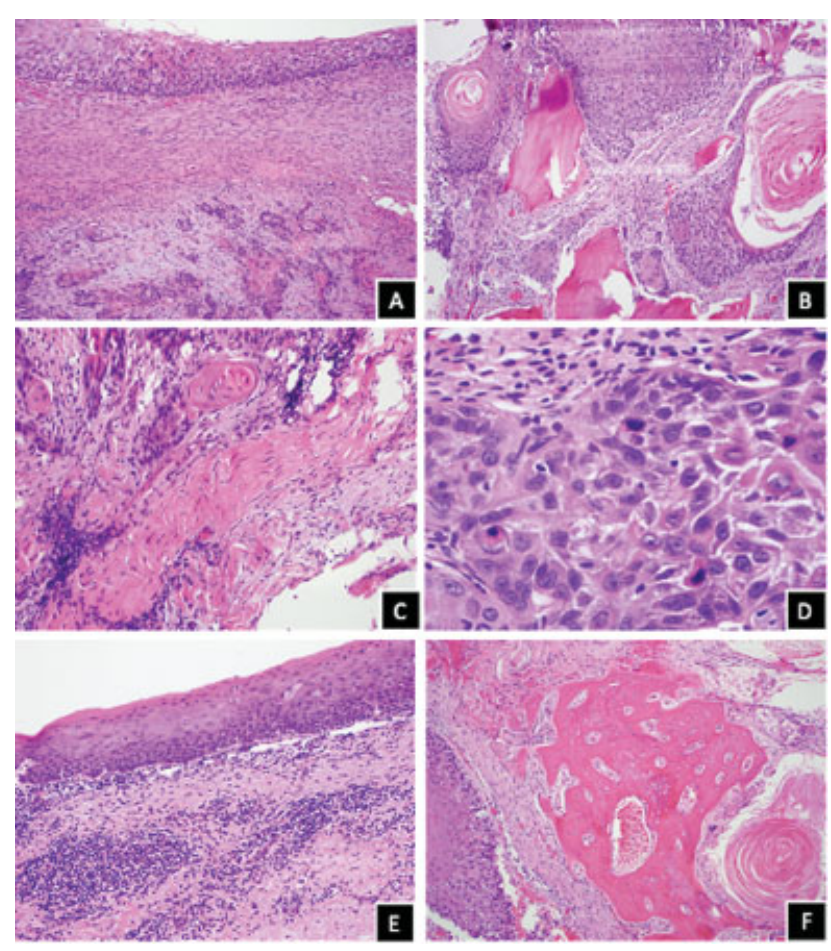

Fig. 1 Invasive keratinizing squamous cell carcinoma (A-D) with background cholesteatoma (E-F). Microscopic examination showing keratinizing squamous cell carcinoma with invasive nests of neoplastic cells surrounded by desmoplastic stromal reaction (A, hematoxylin and eosin [H\&E] at 10x), temporal bone invasion (B, H\&E at 10x), and perineural invasion (C, H\&E at 20x). The carcinoma was moderately differentiated with pleomorphic nuclei and numerous dyskeratinocytes (D, H\&E at 60x). Adjacent to the invasive component there is a background of squamous epithelium, fibrosis, chronic inflammation, and flakey keratin with foci of dysplasia and bone destruction (E, H\&E at 10x).

the most common is SCC, comprising $80 \%$ of all malignancies in this location. ${ }^{8}$

Patients with SCCTB often present between 55 and 65 years of age. ${ }^{9}$ There is a reported female predominance in cancers of the external auditory canal, in contrast to those of the external ear pinna, which shows a male predominance. ${ }^{3,9}$ Symptoms of SSCTB are relatively nonspecific, with chronic otorrhea, otalgia, hearing loss, and bleeding dominating the clinical picture. ${ }^{2,3,6}$ The overlapping differential diagnosis for these nonspecific symptoms contributes to the difficulty and a delay in diagnosis often encountered in SCCTB.

Diagnostic imaging is paramount in the evaluation of chronic otorrhea or other chronic ear symptoms because it cannot only help clarify diagnosis; if malignant, it can lead to earlier diagnosis and guide staging. ${ }^{8}$ The extent of the disease is determined by the Pittsburgh staging system, which is based on location and degree of bone invasion at time of presentation. ${ }^{10}$ However, other mass-like lesions in the ear, including cholesteatomas, may cause bone destruction and mimic malignancy. Imaging can be useful to differentiate this benign process from a more malicious one. Cholesteatomas do not enhance with gadolinium on $\mathrm{T} 1$, whereas most tumors 


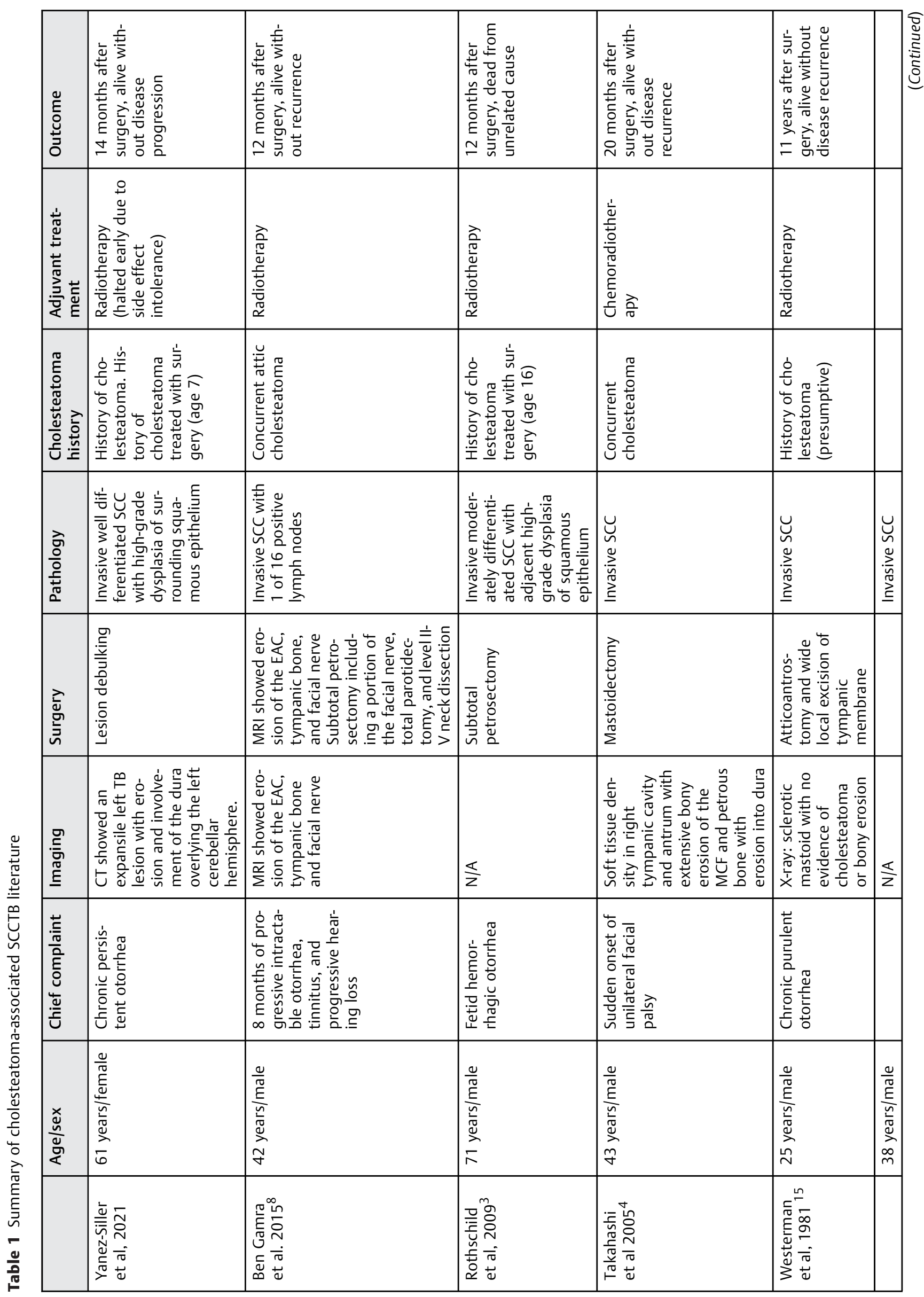


will enhance with gadolinium. Furthermore, cholesteatomas will show high intensity of diffusion weighted images compared with other tumors. ${ }^{8}$ Contrast-enhanced MRI can demonstrate perineural disease and/or dural involvement that would be seen in a malignant and not a benign condition. ${ }^{1,6}$

SCC is the most common malignancy to involve the TB, followed by basal cell carcinoma and adenoid cystic carcinoma. ${ }^{1}$ SCCTB can be primary or secondary. Primary SCCTB is most common (60-80\%), arising from SCC of the EAC and/or the middle ear. ${ }^{11}$ Secondary SCCTB is more uncommon, often as an infiltrating tumor from periauricular skin or salivary glands. Histologically, the tumors of the ear are classified according to the 2017 fourth edition of the World Health Organization Classification of Tumors of the Head and Neck. ${ }^{12}$ SCC is the most common malignancy of the ear. Those arising from the pinna have a different pathogenesis and prognosis compared with those arising within the EAC, which are most common, and middle ear, which are much less common. ${ }^{11}$

The etiology of primary SCCTB from the ear canal is likely multimodal. Ionizing radiation, often secondary to radiotherapy for nasopharyngeal tumors, is an extremely rare but widely accepted risk factor, with a rate $1000 x$ greater than the general population. ${ }^{9}$ However, risk factors related to chronic inflammation, for instance associated with trauma, infection, or surgery to the EAC or middle ear, are more common but controversial. ${ }^{13}$ Importantly, cholesteatoma, a relatively common form of chronic otitis, has also been implicated in the development of primary SCCTB. ${ }^{14} \mathrm{~A}$ total of six cases (five from the literature in addition to the current case report) of SCCTB associated with cholesteatomas are reported in the literature (- Table $\mathbf{1}$ ). $3,4,8,15,16$

Several mechanisms of how cholesteatoma may progress to SCCTB have been proposed, with most believed to result from localized chronic inflammation. ${ }^{2}$ Chronic inflammation is an accepted and well-known risk factor for SCC in many parts of the body. Infection is commonly implicated as the cause of inflammation. This can be caused by certain bacteria, such as antibiotic-resistant and/or biofilm-forming pathogens, particularly Pseudomonas aeruginosa, which can induce squamous cell proliferation. ${ }^{17}$ Additionally in addition to bacteria, a few reports have considered HPV infection as a possible cause of cholesteatoma. ${ }^{18-20} \mathrm{HPV}$ has also been reported in middle ear carcinomas. ${ }^{21,22} \mathrm{At}$ the molecular level, common to other malignancies, alterations in the cell cycle, namely, uninhibited telomerase activity, inactivation of tumor suppressors, and upregulation of growth factor receptors, are thought to govern the progression toward SCCTB. ${ }^{2,23}$ Despite a variety of theories, a direct etiologic and pathophysiologic association between these two TB disease entities remains elusive.

Traditionally, the standard treatment includes surgical excision with or without TB resection, followed by adjuvant radiotherapy, with a recommended total dose of 50 to $70 \mathrm{~Gy} .{ }^{8}$ Chemotherapy is not a part of standard treatment as the occurrence of metastases is uncommon. ${ }^{13}$ By contrast, a few publications have reported primary radiation as an adequate alternative, reporting recurrence and survival rates similar to 
a combined (i.e., surgery plus radiation) approach. ${ }^{2}$ Multidisciplinary approaches employing extensive surgical excision and adjuvant chemoradiotherapy have been proposed with varying results. ${ }^{4,6}$ Others suggest reserving chemoradiotherapy for settings where surgery cannot be performed. ${ }^{13}$ Given the scarcity of studies, which have been primarily limited to small case series without control subjects, a general consensus on the optimal strategy remains to be reached and management approaches vary widely between institutions and authors. ${ }^{2}$

Overall, long-term survival for SCCTB is rare and directly related to the age at presentation. ${ }^{12}$ The prognosis for SCCTB using the University of Pittsburgh staging system has a 2year survival of 95 to $100 \%$ in T1 and T2 patients; unfortunately, tumors are often aggressive, destructive, and diagnosed at later stages, which have a much more dismal prognosis, with a 2 -year survival of $50 \%$ in $\mathrm{T} 3$ and $15 \%$ in T4 patients. ${ }^{14}$ This results not only from its aggressive nature but also from frequent delayed diagnosis resulting in late-stage disease at presentation. Additionally, the proximity to critical neurovascular structures complicates surgical management. ${ }^{2}$

Our patient had undergone surgery for the removal of cholesteatoma, more than 50 years prior to presenting for evaluation at our department, with associated decade-long nonspecific symptoms resembling a chronic inflammatory ear condition, and a TB lesion suspicious of recurrent cholesteatoma. Despite being a benign condition, the propensity for cholesteatomas to recur and to invade bone often mimics a malignancy. Ironically for this reason, true malignancy may go under- or misdiagnosed in this particular area, as presented here, were biopsy of the lesion was consistent with SCC.

Our findings suggest an etiologic association between cholesteatoma and SCCTB, something strongly previously speculated in several prior published reports. ${ }^{3-5}$ Although firm conclusions remain to be drawn, given the overlap in clinical presentation, consideration of the etiologic relationship is warranted in patients with recurrent, persistent, or worsening otologic symptoms, associated with long past history of cholesteatoma with or without prior surgical management.

\section{Conclusion}

The present case report supports the theory that SCCTB may arise from cholesteatoma. ${ }^{2}$ Only a few similar cases have been reported in the literature. ${ }^{3-5,8,15,16} \mathrm{~A}$ high index of suspicion should be employed in patients with a prior history of cholesteatoma and evidence of a TB mass and persistent otologic symptoms. Further research is warranted to elucidate an etiologic relationship among both disease entities.

\section{Note}

Portions of this work were presented as a poster presentation at the 30th Annual North American Skull Base Society Meeting, San Antonio, Texas, February 7-9, 2020.

\section{Disclosure}

The authors have no personal, financial, or institutional interest to declare.

\section{Conflict of Interest}

None declared.

\section{References}

1 Gidley PW, Roberts DB, Sturgis EM. Squamous cell carcinoma of the temporal bone. Laryngoscope 2010;120(06):1144-1151

2 Kenyon GS, Marks PV, Scholtz CL, Dhillon R. Squamous cell carcinoma of the middle ear. A 25-year retrospective study. Ann Otol Rhinol Laryngol 1985;94(03):273-277

3 Rothschild S, Ciernik IF, Hartmann M, Schuknecht B, Lütolf UM, Huber AM. Cholesteatoma triggering squamous cell carcinoma: case report and literature review of a rare tumor. Am J Otolaryngol 2009;30(04):256-260

4 Takahashi K, Yamamoto Y, Sato K, Sato Y, Takahashi S. Middle ear carcinoma originating from a primary acquired cholesteatoma: a case report. Otol Neurotol 2005;26(01):105-108

5 Kleinsasser O, Schulze W, Glanz H. [Squamous cell carcinoma of the middle ear following tympanoplasty]. HNO 1984;32(02): 61-62

6 Lovin BD, Gidley PW. Squamous cell carcinoma of the temporal bone: a current review. Laryngoscope Investig Otolaryngol 2019; 4(06):684-692

7 Moody SA, Hirsch BE, Myers EN. Squamous cell carcinoma of the external auditory canal: an evaluation of a staging system. Am J Otol 2000;21(04):582-588

8 Ben Gamra O, Abid W, Nacef I, et al. Cholesteatoma associated with squamous cell carcinoma of the external auditory canal: case report and literature review. Egypt J Ear, Nose Throat Allied Sci 2015;16(03):269-274

9 Thompson LDR. Update From the 4th Edition of the World Health Organization Classification of Head and Neck Tumours: tumours of the ear. Head Neck Pathol 2017;11(01):78-87

10 Arriaga M, Curtin H, Takahashi H, Hirsch BE, Kamerer DB. Staging proposal for external auditory meatus carcinoma based on preoperative clinical examination and computed tomography findings. Ann Otol Rhinol Laryngol 1990;99(9 Pt 1): 714-721

11 Ungar OJ, Santos F, Nadol JB, et al. Invasion patterns of external auditory canal squamous cell carcinoma: a histopathology study. Laryngoscope 2021;131(02):E590-E597

12 Weltgesundheitsorganisation. WHO Classification of head and Neck Tumours. 4th edition. (Naggar AK el-, Chan JKC, Grandis JR, Takata T, Slootweg PJ, eds.). International Agency for Research on Cancer; 2017

13 Yin M, Ishikawa K, Honda K, et al. Analysis of 95 cases of squamous cell carcinoma of the external and middle ear. Auris Nasus Larynx 2006;33(03):251-257

14 Allanson BM, Low TH, Clark JR, Gupta R. Squamous cell carcinoma of the external auditory canal and temporal bone: an update. Head Neck Pathol 2018;12(03):407-418

15 Westerman ST, Sylvia LC, Tepper E. Carcinoma arising out of a primary acquired cholesteatoma. J Med Soc N J 1981;78(09):600-602

16 Coachman EH. Squamous cell carcinoma secondary to cholesteatoma. AMA Arch Otolaryngol 1951;54(02):187. Doi: 10.1001/ archotol.1951.03750080075011

17 Kuo CL. Etiopathogenesis of acquired cholesteatoma: prominent theories and recent advances in biomolecular research. Laryngoscope 2015;125(01):234-240

18 Bergmann K, Hoppe F, He Y, et al. Human-papillomavirus DNA in cholesteatomas. Int J Cancer 1994;59(04):463-466 
e18 SCCTB Arising from Cholesteatoma Yanez-Siller et al.

19 Chao WY, Chang SJ, Jin YT. Detection of human papillomavirus in cholesteatomas. Eur Arch Otorhinolaryngol 2000;257(03):120-123

20 Malagutti N, Rotondo JC, Cerritelli L, et al. High human papillomavirus DNA loads in inflammatory middle ear diseases. Pathogens 2020;9(03):1-10

21 Jin YT, Tsai ST, Li C, et al. Prevalence of human papillomavirus in middle ear carcinoma associated with chronic otitis media. Am J Pathol 1997;150(04):1327-1333
22 Tsai ST, Li C, Jin YT, Chao WY, Su IJ. High prevalence of human papillomavirus types 16 and 18 in middle-ear carcinomas. Int J Cancer 1997;71(02):208-212

23 Watabe-Rudolph M, Rudolph KL, Averbeck T, Buhr T, Lenarz T, Stöver T. Telomerase activity, telomere length, and apoptosis: a comparison between acquired cholesteatoma and squamous cell carcinoma. Otol Neurotol 2002;23(05):793-798 